\title{
Modified Gompertz equation for electrotherapy murine tumor growth kinetics: predictions and new hypotheses
}

Luis E Bergues Cabrales ${ }^{2,1^{*}}$, Juan J Godina Nava², Andrés Ramírez Aguilera ${ }^{3}$, Javier A González Joa ${ }^{4}$, Héctor M Camué Ciria ${ }^{1}$, Maraelys Morales González ${ }^{5}$ Miriam Fariñas Salas ${ }^{1}$, Manuel Verdecia Jarque ${ }^{6}$, Tamara Rubio González", Miguel A O’Farril Mateus ${ }^{8}$, Soraida C Acosta Brooks ${ }^{9}$, Fabiola Suárez Palencia', Lisset Ortiz Zamora ${ }^{5}$, María C Céspedes Quevedo ${ }^{8}$, Sarah Edward Seringe ${ }^{8}$, Vladimir Crombet Cuitié ${ }^{1}$, Idelisa Bergues Cabrales ${ }^{4}$, Gustavo Sierra González ${ }^{10}$

\begin{abstract}
Background: Electrotherapy effectiveness at different doses has been demonstrated in preclinical and clinical studies; however, several aspects that occur in the tumor growth kinetics before and after treatment have not yet been revealed. Mathematical modeling is a useful instrument that can reveal some of these aspects. The aim of this paper is to describe the complete growth kinetics of unperturbed and perturbed tumors through use of the modified Gompertz equation in order to generate useful insight into the mechanisms that underpin this devastating disease.

Methods: The complete tumor growth kinetics for control and treated groups are obtained by interpolation and extrapolation methods with different time steps, using experimental data of fibrosarcoma Sa-37. In the modified Gompertz equation, a delay time is introduced to describe the tumor's natural history before treatment. Different graphical strategies are used in order to reveal new information in the complete kinetics of this tumor type.

Results: The first stage of complete tumor growth kinetics is highly non linear. The model, at this stage, shows different aspects that agree with those reported theoretically and experimentally. Tumor reversibility and the proportionality between regions before and after electrotherapy are demonstrated. In tumors that reach partial remission, two antagonistic post-treatment processes are induced, whereas in complete remission, two unknown antitumor mechanisms are induced.

Conclusion: The modified Gompertz equation is likely to lead to insights within cancer research. Such insights hold promise for increasing our understanding of tumors as self-organizing systems and, the possible existence of phase transitions in tumor growth kinetics, which, in turn, may have significant impacts both on cancer research and on clinical practice.
\end{abstract}

\section{Background}

Tumors are complex biological systems, and, in spite of great therapeutic advances, many of these still do not respond to treatment and lead to death. Part of the complexity of the problem is the sheer consequence of

\footnotetext{
* Correspondence: berguesc@yahoo.com

${ }^{2}$ Departamento de Física, Centro de Investigación y Estudios Avanzados del Instituto Politécnico Nacional, Laboratorio de Estimulación Magnética, Av. Instituto Politécnico Nacional 2508, Col. San Pedro Zacatenco, Ap. Post. 14740, México, D.F. 07000, México 07360, Distrito Federal, México

Full list of author information is available at the end of the article
}

the tumor size and its histogenic characteristics. The cancer phenomenon continues to challenge oncologists. The pace of progress has often been slow, in part because of the time required to evaluate new therapies. To reduce the time to approval, new paradigms for assessing therapeutic efficacy are needed [1]. This requires the intellectual energy of scientists working in the field of mathematics and physics, collaborating closely with biologists and clinicians. This essentially means that the heuristic experimental approach, which is the traditional investigative method in the biological

\section{Biomed Central}


sciences, should be complemented by a mathematical modeling approach [2].

Significant research has been done in the modeling of tumors using theoretical models and computer simulations in order to describe and predict various aspects of tumor growth kinetics (TGK). Predicting tumor growth is important in the planning and evaluation of screening programs, clinical trials, and epidemiological studies, as well as in the adequate selection of dose-response relationships regarding the proliferative potential of tumors [2-5].

The biological behavior of a malignant tumor is highly influenced by its growth rate, which is determined by many intratumoral and micro-environmental factors. The space-time permanent growth is probably the most characteristic feature of a malignant tumor.

Further advancement in mathematical modeling of TGK critically depends on a thorough testing of proposed models against new data as they become available with the development of experimental techniques [3-6]. Thus, it is apparent that theoretical mathematical models are needed to study cancer.

In electrotherapy (ET) with low-level direct electrical current (DEC), mathematical modeling has not been used. ET was revolutionary when first introduced and is a promising surgical technique for destroying tumors. It has been shown to be a very useful, alternative tool against cancer. Preclinical and clinical studies have shown that ET is simple, safe, effective, and, minimally traumatic, with few side effects. It provides a method for treating solid cancers that are conventionally inoperable, those that cannot be resected after thoracotomy, and those that are not responsive to chemotherapy or radiotherapy [7-10]. Similar results have been reported by our research group [11-15].

Although preclinical and clinical studies have shown that ET has a marked antitumor effect, it is not widely used in clinical practice. The reason is that ET is not a well-established therapy due to the lack of a standardized method and unclear knowledge concerning the mechanisms involved. As a result an optimal electrode distribution has not been determined for ET, nor has the dose-response relationship been established. For this reason, we pay special attention to these two factors $[16,17]$.

Camué et al. [14] experimentally report that an increase in DEC intensity increases its antitumor effectiveness, and that Ehrlich and fibrosarcoma Sa-37 tumors have a DEC threshold for which their complete remission is reached. These results have been theoretically corroborated through the use of a modified Gompertz equation (MGE), which has a good prediction capability to describe both unperturbed and DECperturbed

\section{TGK [17].}

Many intrinsic processes that occur in both unperturbed and DEC-perturbed TGK are unknown. We believe that the MGE can be used as a tool to reveal some of these processes in order to improve DEC effectiveness. The aim of this paper is to describe the complete growth kinetics of unperturbed and DECperturbed fibrosarcoma Sa-37 tumors through the MGE in order to generate useful insights into the mechanisms that underpin this devastating disease. In this study, we analyze this model taking into account the experimental data reported in [14] for fibrosarcoma Sa-37 tumor. Also, we discuss the current limitations and potential implications of this model for further TGK research. It is important to note that the results reported in [14] and [17] support this paper.

\section{Methods}

This study is approved by the Committees of Ethics of the National Center of Electromagnetism Applied (CNEA) and the Conrado Benitez Oncologic hospital, Santiago de Cuba, Cuba.

\section{Complete growth kinetics for unperturbed and DEC- perturbed tumors}

Our experiences in preclinical and clinical studies have indicated that DEC-treated TGK is complex, with two well-defined regions (REG-I and REG-II). REG-I (defined before DEC treatment is performed) includes the initial time of tumor cell inoculation ( $t=0$ days) up to the moment that tumor is perturbed by a DEC stimulus, which occurs when it reaches a volume $V_{o}$ (initial volume selected by the therapist). REG-II (defined after DEC treatment is performed) includes the time at which the tumor is perturbed by DEC stimulus up to the end of the experiment.

In preclinical studies, the end of the experiment is fixed by the researcher, whereas in clinical studies, it can occur at multiple events: 1) the patient dies, 2) the patient leaves the clinical trial, or 3) the patient is completely cured $[9,10,15]$. It is important to point out that this REG-II is only reported in the field of ET in cancer [9-15,18-21].

\section{Electrochemical treatment}

Once fibrosarcoma Sa-37 tumors have reached approximately $\mathrm{V}_{\mathrm{o}}=0.5 \mathrm{~cm}^{3}$ in $\mathrm{BALB} / \mathrm{c}$ mice, four platinum electrodes are inserted into their bases and a single-shot electrotherapy is supplied. $V_{\mathrm{o}}$ is reached 15 days after viable tumor cells are inoculated in the dorsolateral region of the animals. Four groups (one control group and three treated groups), each consisting of ten mice, were randomly formed: the control group (CG), a 
treated group with $36 \mathrm{C} / \mathrm{cm}^{3}\left(18 \mathrm{C}\right.$ in $\left.0.5 \mathrm{~cm}^{3}\right)$ and 6.7 $\mathrm{mA}$ for $45 \mathrm{~min}$ (TG1), a treated group with $63 \mathrm{C} / \mathrm{cm}^{3}$ $\left(31.5 \mathrm{C}\right.$ in $0.5 \mathrm{~cm}^{3}$ ) and $11.7 \mathrm{~mA}$ for $45 \mathrm{~min}$ (TG2), and a treated group with $80 \mathrm{C} / \mathrm{cm}^{3}\left(40.0 \mathrm{C}\right.$ in $\left.0.5 \mathrm{~cm}^{3}\right)$ and $14.8 \mathrm{~mA}$ for $45 \mathrm{~min}$ (TG3). The experimental details are discussed by Camué et al. [14].

\section{Modified Gompertz equation}

A feature of the MGE is that it is developed after the experiments. It is implemented in order to fit the experimental data corresponding to REG-II for the Ehrlich and fibrosarcoma Sa-37 TGK [17], given by

$$
V^{*}(t)=V_{o} e^{\left(\frac{\alpha^{*}}{\beta}\right)\left(1-e^{-\beta t}\right)}
$$

where

$$
\alpha^{*}=\left[a_{1}\left(1-e^{-\gamma t}\right)+a_{2}\right] \alpha,
$$

with

$$
a_{1}=\left(\frac{i}{i_{o}}\right)\left(2-\frac{i}{i_{o}}\right),
$$

and

$$
a_{2}=\left(1-\frac{i}{i_{o}}\right),
$$

All parameters involved are real and positive. $\mathrm{V}^{*}\left(\mathrm{t}^{\prime}\right)$ represents the tumor volume (TV) at time $t^{\prime}$ after DEC treatment. The parameter $\alpha(\alpha>0)$ is the intrinsic growth rate of the tumor related to the initial mitosis rate. The parameter $\beta(\beta>0)$ is the growth deceleration factor related to the anti-angiogenic process. The parameter $\alpha^{*}$ is the modified tumor growth rate due to DEC action. $i(i>0)$ is the DEC intensity that flows through the tumor by the application of an external electric field. $i_{o}\left(i_{o}>0\right)$ is the polarization current (or electric current distributed into the tumor by DEC action). The parameter $\gamma$ is the first-order exponential decay rate of the net effect induced in the solid tumor after the DEC is removed and its inverse is the decay constant (or decay time) that characterizes the duration of such an effect. $a_{1}$ and $a_{2}$ are dimensionless parameters that depend only on the $\left(i / i_{o}\right)$ ratio.

The results obtained from fitting the experimental data for REG-II of fibrosarcoma Sa-37 TGK for CG, TG1, TG2, and TG3 are shown in Table 1. The numerical information is obtained upon fitting the individual tumor growth data and then fitting the data for each experimental group. The mean values and standard
Table 1 Mean \pm standard error of the parameters obtained from fitting the experimental data of the growth curve of fibrosarcoma Sa-37 tumors using the MGE

\begin{tabular}{ccccc}
\hline Groups $^{*}$ & $\boldsymbol{\alpha}\left(\right.$ days $\left.^{-1}\right)$ & $\boldsymbol{\beta}\left(\right.$ days $\left.^{-\mathbf{1}}\right)$ & $\boldsymbol{\gamma}\left(\right.$ days $\left.^{-\mathbf{1}}\right)$ & $\boldsymbol{i}_{\boldsymbol{o}}(\mathbf{m A})$ \\
\hline CG & $0.513 \pm 0.009$ & $0.262 \pm 0.006$ & $0.000 \pm 0.000$ & $0.000 \pm 0.000$ \\
TG1 & $1.793 \pm 0.028$ & $0.142 \pm 0.006$ & $0.184 \pm 0.003$ & $4.342 \pm 0.007$ \\
TG2 & $1.584 \pm 0.030$ & $0.076 \pm 0.002$ & $0.107 \pm 0.001$ & $4.342 \pm 0.007$ \\
TG3 & $0.006 \pm 0.001$ & $0.207 \pm 0.002$ & $0.189 \pm 0.016$ & $1.080 \pm 0.210$
\end{tabular}

*CG: control group. TG1: treated group with electrical current of $6.7 \mathrm{~mA}$. TG2: treated group with $11.7 \mathrm{~mA}$. TG3: treated group with $14.8 \mathrm{~mA}$. Each experimental group consisted of 10 mice $[14,17]$, which are exposed for 45 minutes.

errors of these optimized parameters after fitting were computed [17].

\section{Interpolation of data corresponding to REG-II of fibrosarcoma Sa-37 TGK}

Interpolation of experimental data corresponding to REG-II of fibrosarcoma Sa-37 TGK was developed. From an experimental point of view, to perform such a study, the information in ET is reported in terms of a non equidistant time dependence of TV $\left(\mathrm{V}^{*}\left(\mathrm{t}^{\prime}\right)\right.$ vs. $\mathrm{t}^{\prime}$ plot, named TV plot) spaced by one day or more. As a result, the TGK details are not revealed. At the experimental level, it is difficult to show the TGK for a small time step like one day because such a study would be cumbersome, expensive in resources, timeconsuming, and requiring excessive handling of animals, which is not permitted by the ethics code care and use of Laboratory Animals Committee. For this reason, we interpolated the experimental data corresponding to REG-II for this tumor type using different time steps, $\Delta \mathrm{t}(1,1 / 3,1 / 8,1 / 24$, and $1 / 48$ days). In this case, we take into account the mean values of each parameter in the MGE for each experimental group (Table 1).

\section{Reconstruction of REG-I for fibrosarcoma Sa-37 TGK}

In the ET framework, neither experimental nor theoretical reports have taken into account REG-I of TGK which, for the former, can be very important for understanding the fibrosarcoma Sa-37 natural history before DEC treatment and its future influence on therapeutic effectiveness after DEC treatment. For this reason, we reconstructed this first region using an extrapolation method (to find unknown values for TV in points that are outside the typical studied range) for each $\Delta t$. In order to obtain the complete TGK for CG, we substitute $\mathrm{t}^{\prime}$ with $(\mathrm{t}-\tau)$ in Equation 1, keeping in mind the $\alpha, \beta$, and $\mathrm{V}_{\mathrm{o}}$ parameters (Table 1 ) and the interpolated experimental data for REG-II. In this case, $\tau$ is a time delay 
that represents the time interval from the point at which the tumor cells are inoculated in the host until the solid tumor reaches $\mathrm{V}_{\mathrm{o}}$.

The considerations included in the MGE are:

1. For unperturbed tumors $\left(\alpha^{*}=\alpha\right.$ for $\left.i=0\right), \alpha$ in Equation 1, is constant during TGK. In this case, the MGE coincides with the conventional Gompertz equation $[1,17,22,23]$.

2. REG-I for fibrosarcoma Sa-37 TGK is the same for CG, TG1, TG2, and TG3. As a result, $\alpha$ in Equation 1, is the same for all of the experimental groups. This assumption has been experimentally corroborated, since the tumors in CG, TG1, TG2, and TG3 reach $V_{\mathrm{o}}$ at approximately the same time $\tau(\tau=15$ days) [14].

The MGE can be rewritten in the form

$$
V^{*}(t)= \begin{cases}V_{o} e^{\left(\frac{\alpha}{\beta}\right)\left(1-e^{-\beta(t-\tau)}\right)} & 0 \leq t \leq \tau \\ V_{o} e^{\left(\frac{\alpha}{\beta}\right)\left(1-e^{-\beta(t-\tau)}\right)} & \tau \leq t \leq \tau+t^{\prime},\end{cases}
$$

where $t$ is the time that elapses from the initial moment at which tumor cells are inoculated in the host $(t=0$ days $)$ up to the end of the experiment. $t^{\prime}$ is the time that elapses from the moment of DEC application up to the end of the experiment.

\section{Graphical strategies for the analysis of TGK of the experimental groups}

Different graphical strategies are used in order to obtain further time-dependent information for both untreated and DEC-treated TGK that is not revealed in a simple TV plot. For this reason, we use the following plots: first derivative of tumor volume (FDTV) versus $t$, named the FDTV plot (or $d V^{*}(t) / d t$ vs. $t$ plot); TV dependence of FDTV, named FDTV-TV plot (or dV*(t)/dt vs. $\mathrm{V}^{*}(\mathrm{t})$ plot), the time consecutive dependence on TV plot, named CTV plot (or $\mathrm{V}^{*}(\mathrm{t})$ vs. $\mathrm{V}^{*}(\mathrm{t}-\Delta \mathrm{t})$ plot); and the modules and loglog plots for TV and FDTV-TV in order to analyze whether REG-I and REG-II for TG3 are the same.

It is important to point out that the results shown in this paper are in long format (scaled fixed point with 15 digits after the decimal point).

\section{Results}

\section{Analysis of complete unperturbed fibrosarcoma Sa-37 TGK}

The complete growth kinetics of unperturbed fibrosarcoma Sa-37 tumors are generated by interpolation of the experimental data for REG-II and the extrapolation process for REG-I using Equation 5 with values for $\alpha, \beta$, $\tau$, and $V_{o}$ from the CG (Table 1). TGK exhibits a

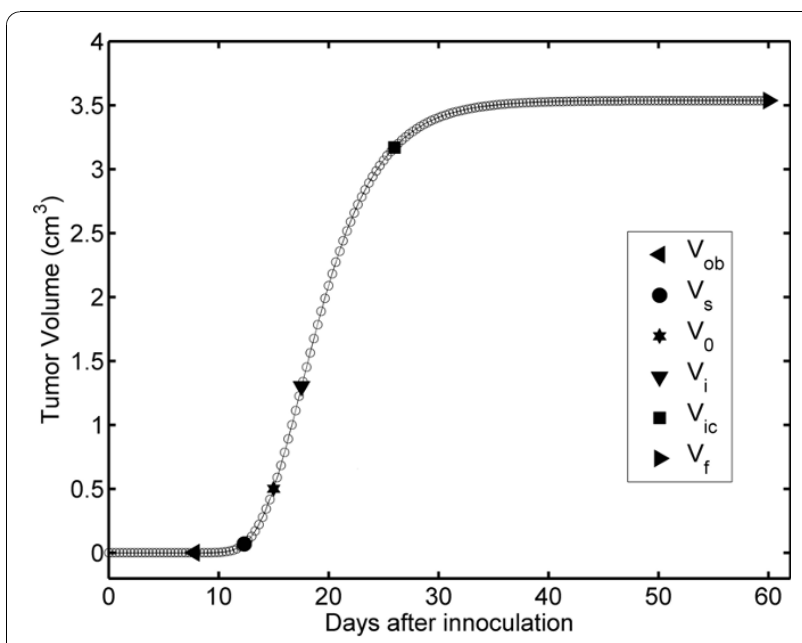

Figure 1 Time dependence of TV: Unperturbed fibrosarcoma Sa-37 TGK (CG) for the parameters $i=0 \mathrm{~mA}, \alpha=0.513$ days $^{-1}$, $\beta=0.262$ days $^{-1}, V_{o}=0.5 \mathrm{~cm}^{3}$ and a time step of $\Delta t=1 / 3$ days

characteristic S shape with three stages (SI, SII, and SIII), which are well defined for all $\Delta t$ values, as shown in Figure 1 for $\Delta t=1 / 3$ days.

The results show that SI is nonlinear and that there are two intersection points that separate each of the stages. The first point $\left(\mathrm{V}_{\mathrm{s}}\right.$ in Figure 1$)$ is obtained from the interception between SI and SII, and it represents the beginning of SII (TGK is triggered). The second point $\left(\mathrm{V}_{\mathrm{ic}}\right.$ in Figure 1) is obtained by the interception of SII and SIII, representing the beginning of SIII (TV tends to a limit value, $\left.V_{f}\right)$. $V_{\text {ic }}$ represents the irreversible TV from which it growth up to $V_{f}$. The $V_{s}, V_{i c}$, and $V_{f}$ points are characterized by an ordered pair (t, TV) and are estimated as (12.34 days, $\left.0.069 \mathrm{~cm}^{3}\right),\left(25.99\right.$ days, $\left.3.169 \mathrm{~cm}^{3}\right)$ and $(60$ days, $3.536 \mathrm{~cm}^{3}$ ) for all values of $\Delta t$, respectively.

The interpolation and extrapolation processes reveal that unperturbed fibrosarcoma Sa-37 TGK has a point of inflection, $V_{\mathrm{i}}$ at $\left(17.56\right.$ days, $\left.1.301 \mathrm{~cm}^{3}\right)$. This value may also be analytically corroborated by making $i=0$ and setting the second derivative of Equation 1 to zero. $V_{i}$ is a point in the TGK at which the curvature changes from concave upwards (positive curvature) to concave downwards (negative curvature). Additionally, these processes predict three other TV values, which are observed in the experiment: $\mathrm{V}_{\mathrm{o}}=0.5 \mathrm{~cm}^{3}$ at 15 days; $0.02 \mathrm{~cm}^{3}$ at 11.29 days; and $0.03 \mathrm{~cm}^{3}$ at 11.60 days [14]. In preclinical studies, our experience shows that $0.02 \mathrm{~cm}^{3}$ is the smallest measurable TV, designated as $V_{m}$ [11-14]. $V_{m}$ for fibrosarcoma Sa-37 tumors is experimentally observed at 12 days [14]. The difference in time is 0.71 days, which is not significant from an experimental point of view.

We macroscopically observe the first non-zero volume, $\mathrm{V}_{\mathrm{ob}}$, for fibrosarcoma Sa-37 at 8 days (Figure 


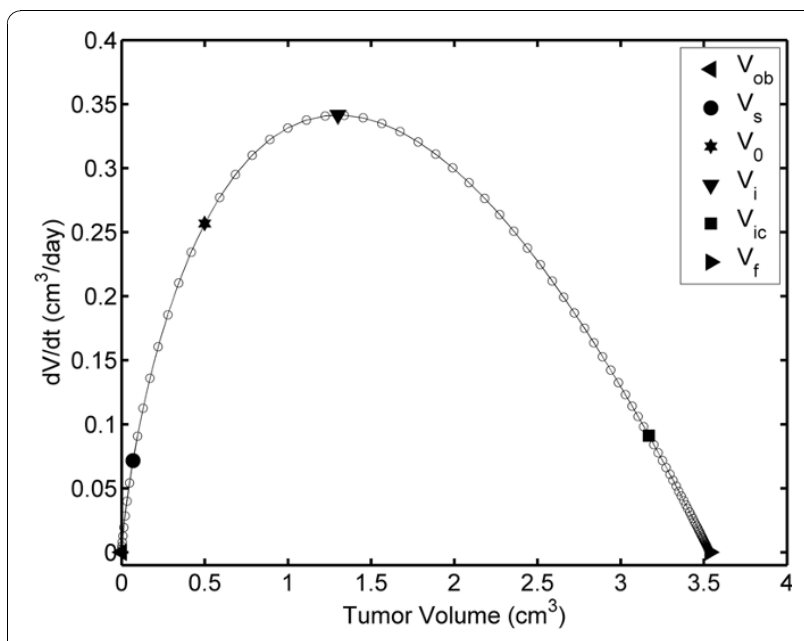

Figure 2 TV dependence of the FDTV: Unperturbed fibrosarcoma Sa-37 TGK (CG) for the parameters $i=0 \mathrm{~mA}, \alpha=0.513$ days $^{-1}$, $\beta=0.262$ days $^{-1}, v_{o}=0.5 \mathrm{~cm}^{3}$ and a time step of $\Delta t=1 / 3$ days.

1). This tumor size is observable and palpable, but not measurable. Equation 5 estimates $V_{\mathrm{ob}}$ to be 0.000016 $\mathrm{cm}^{3}(0.031 \mathrm{~cm}$ in diameter) for this time value.

The FDTV-TV plot (Figure 2) shows that TV increases between $V_{\text {obs }}$ and $V_{f}$; however, FDTV increases from $V_{\mathrm{s}}$ to $\mathrm{V}_{\mathrm{i}}$ and then decreases to zero (from $V_{\mathrm{i}}$ to $\mathrm{V}_{\mathrm{f}}$ ). Figure 2 illustrates that reaches its maximum value, FDTV $V_{\max }$ when TV reaches $\mathrm{V}_{\mathrm{i}}$. The ordered pair $\left(\mathrm{V}_{\mathrm{i}}\right.$, FDTV $\left._{\text {max }}\right)$ is $\left(1.301 \mathrm{~cm}^{3}, 0.341 \mathrm{~cm}^{3} /\right.$ day $)$, which is observed at 17.56 days.

\section{Analysis of REG-II DEC-perturbed TGK for TG2}

We directly present the results for REG-II TGK for tumors treated with DEC for 45 minutes because the REG-I is similar to that of the CG. The complete fibrosarcoma Sa-37 TGK treated with $11.7 \mathrm{~mA}$ (TG2) is shown in Figure 3, for $\Delta t=1 / 3$ days, in agreement with other values of $\Delta t=1,1 / 8,1 / 24$, and $1 / 48$ days (results not shown). This figure reveals that REG-II TGK (from $V_{o}$ up to end of the experiment) is characterized by two sub-regions (REG-IIa and REG-IIb). In REG-IIa, TV decreases from $V_{o}$ to its minimum volume, $V_{\text {min }}$, whereas in REG-IIb, it increases from $V_{\min }$ until the end of the experiment. Additionally, $\mathrm{V}_{\min }$ is estimated by the interception of REG-IIa and REG-IIb, resulting in a value of $0.0698 \mathrm{~cm}^{3}$ that is reached at 20.58 days, for all $\Delta t$ values, as shown in Figure 3, for $\Delta t=1 / 3$ days. $V_{\text {min }}$ is analytically corroborated through the following transcendent equation, constructed by minimizing $\mathrm{V}^{*}\left(\mathrm{t}^{\prime}\right)$ in Equation 1, given by

$$
a_{1} \gamma e^{-\gamma t}+\beta\left(a_{1}+a_{2}\right) e^{-\beta t}-a_{1}(\gamma+\beta) e^{-\gamma t} e^{-\beta t}=0
$$

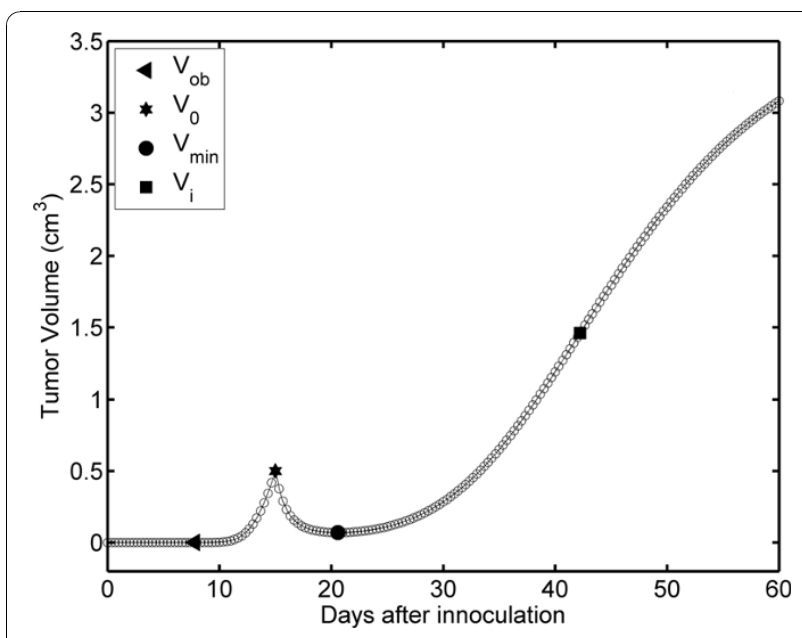

Figure 3 Time dependence of TV: DEC-perturbed fibrosarcoma Sa-37 TGK (TG2) for the parameters $i=11.7 \mathrm{~mA}, \alpha=1.584$ days $^{-1}, \beta=0.076$ days $^{-1}, \gamma=0.107$ days $^{-1}, i_{o}=7.431 \mathrm{~mA}$, and $\mathrm{V}_{\mathrm{o}}=0.5 \mathrm{~cm}^{3}$ and a time step of $\Delta \mathrm{t}=1 / 3$ days.

For this, we substitute the values of $\alpha, \beta, \gamma, i$, and $i_{o}$ for TG2 (Table 1) in Equation $6 . \mathrm{V}_{\text {min }}$ is experimentally observed to be $0.07 \mathrm{~cm}^{3}$, reached at 21 days after the inoculation process. The differences between the estimated and analytical values are $0.0002 \mathrm{~cm}^{3}$ for TV and 0.42 days for time, neither of which are significant at the experimental level.

Figure 4 shows that when TV decreases from $V_{o}$ to $\mathrm{V}_{\text {min }}$, FDTV first decreases from 0.5 to $0.376 \mathrm{~cm}^{3}$ (positive slope) and then it increases from 0.376 to 0.069 $\mathrm{cm}^{3}$ (negative slope). The FDTV values for 0.376 and $0.069 \mathrm{~cm}^{3}$ are $-0.3069 \mathrm{~cm}^{3} /$ days (at 15.33 days) and $0.000068 \mathrm{~cm}^{3} /$ days (at 20.58 days), respectively. The

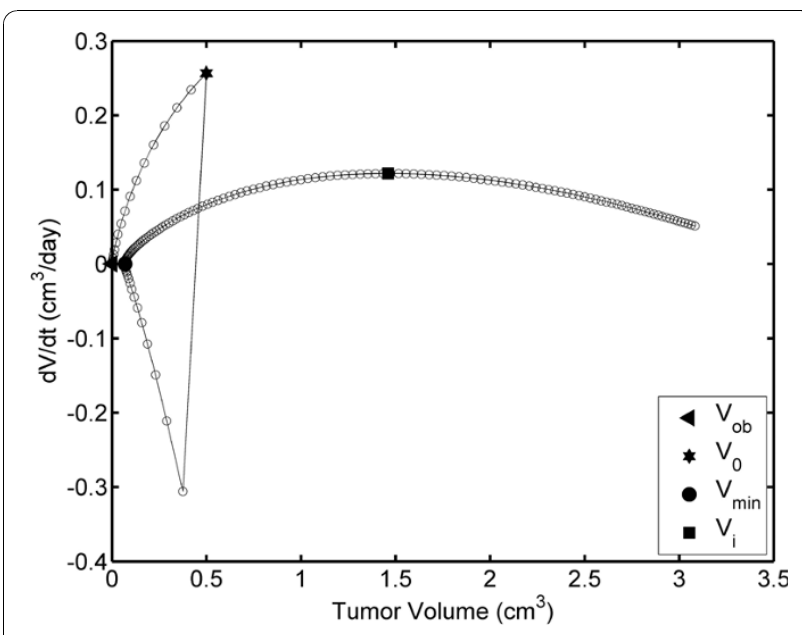

Figure 4 TV dependence of the FDTV: DEC-perturbed fibrosarcoma Sa-37 TGK (TG2) for the parameters $i=11.7 \mathrm{~mA}$, $\alpha=1.584$ days $^{-1}, \beta=0.076$ days $^{-1}, \gamma=0.107$ days $^{-1}, i_{o}=7.431$ $\mathrm{mA}$, and $\mathrm{V}_{\mathrm{o}}=0.5 \mathrm{~cm}^{3}$ and a time step of $\Delta \mathrm{t}=1 / 3$ days. 
values of $-0.3069 \mathrm{~cm}^{3} /$ days corresponds to the minimum negative value of FDTV, FDTV $\mathrm{Fin}_{\text {min }}$, which is observed at 15.33 days and between $V_{o}$ and $V_{\text {min }}$ (Figure 4); however, this is not revealed in the TV plot (Figure 3). The TV correspondent to FDTV min $_{\text {in }}$ in Figure 4, reveals that when the TV reaches $V_{\text {min }}$, it always increases from $V_{\text {min }}$ up to $V_{f}$; however, FDTV increases from $V_{\text {min }}$ up to $\mathrm{V}_{\mathrm{i}}$ (predicted as $\left(1.461 \mathrm{~cm}^{3}, 0.121 \mathrm{~cm}^{3} /\right.$ days) and reached at 42.22 days). Then, FDTV decreases to zero from $V_{i}$ up to $\mathrm{V}_{\mathrm{f}}$.

\section{Analysis of REG-II DEC-perturbed TGK for TG3}

A completely different picture is observed in REG-II of the DEC-perturbed fibrosarcoma Sa-37 TGK for TG3, as shown in Figure 5 . This TGK is characterized by a decrease of TV from $V_{o}$ to $0 \mathrm{~cm}^{3}$, with two well-defined sub-regions (REG-IIc and REG-IId). In REG-IIc, TV rapidly changes from $V_{o}$ to $T V$ from which begins its complete destruction, named $\mathrm{V}_{\mathrm{d}}$ (estimated as 0.014 $\mathrm{cm}^{3}$ at 21.58 days); however, in REG-IId, it slowly varies from $V_{d}$ to zero, reached at 30 days after the inoculation process ( 15 days after DEC treatment), as shown in Figure 5 , for $\Delta t=1 / 3$ days. These results are in agreement with those obtained for the other values of $\Delta t$. This figure reveals that in REG-IIc, there is a point of inflection $\mathrm{V}_{\mathrm{id}}$, which is predicted at $\left(16.15\right.$ days, $\left.0.355 \mathrm{~cm}^{3}\right)$.

Figure 6 reveals, that TV and FDTV decrease from $V_{o}$ to $V_{\text {id }}$ (estimated as $0.355 \mathrm{~cm}^{3},-0.163 \mathrm{~cm}^{3} /$ day) at 16.15 days (positive slope), and then both magnitudes decrease up to a state of the tumor characterized by the ordered pair $\left(0.014 \mathrm{~cm}^{3},-0.0072 \mathrm{~cm}^{3} /\right.$ day) at 21.58 days (negative slope). TV and FDTV abruptly decrease to zero for TV smaller than $V_{d}$ (negative slope).

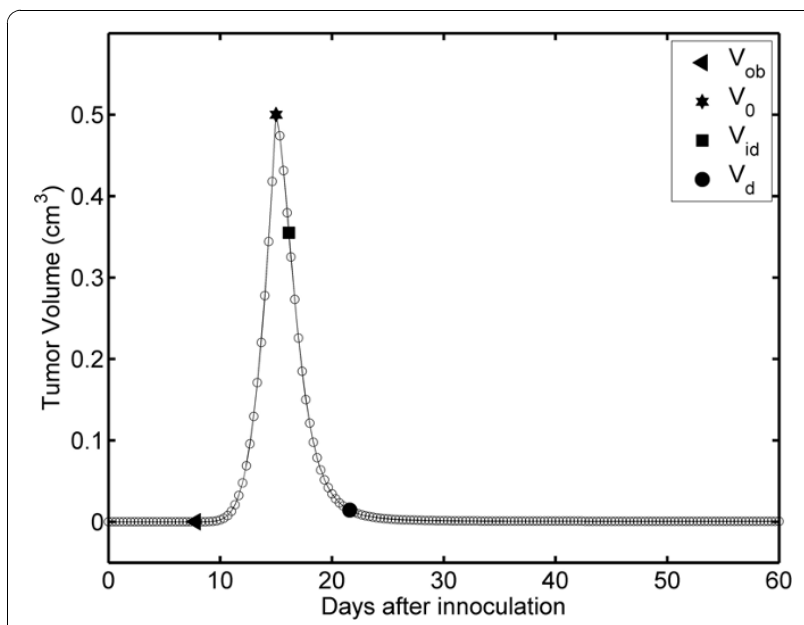

Figure 5 Time dependence of TV: DEC-perturbed fibrosarcoma Sa-37 TGK (TG3) for the parameters $i=14.8 \mathrm{~mA}, \alpha=0.006$ days $^{-1}, \beta=0.207$ days $^{-1}, \gamma=0.189$ days $^{-1}, i_{o}=1.080 \mathrm{~mA}$, and $\mathrm{V}_{\mathrm{o}}=0.5 \mathrm{~cm}^{3}$ and a time step of $\Delta \mathrm{t}=1 / 3$ days

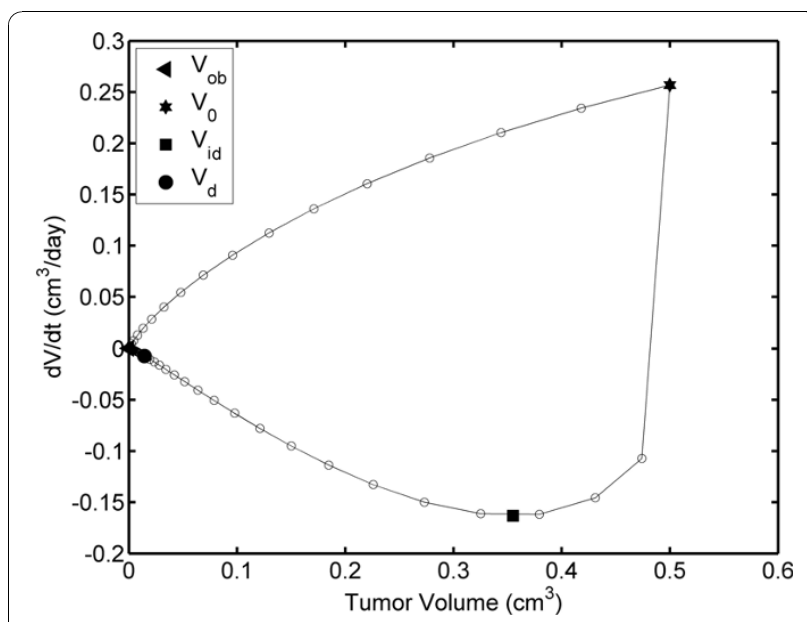

Figure 6 TV dependence of the FDTV: DEC-perturbed fibrosarcoma Sa-37 TGK (TG3) for the parameters $i=14.8 \mathrm{~mA}$, $\alpha=0.006$ days $^{-1}, \beta=0.207$ days $^{-1}, \gamma=0.189$ days $^{-1}, i_{o}=1.080$ $\mathrm{mA}$, and $\mathrm{V}_{\mathrm{o}}=0.5 \mathrm{~cm}^{3}$ and a time step of $\Delta \mathrm{t}=1 / 3$ days.

We propose a CTV plot in order to demonstrate whether the tumor can be completely reversible, as shown in Figure 7. In this figure, a closed loop appears for $\Delta t=1 / 3$ and $1 / 24$ days, being narrower for $\Delta t=$ $1 / 24$ days. This figure reveals that REG-I and REG-II for fibrosarcoma Sa-37 TGK are not symmetric. In addition, an analysis of the FDTV module versus TV plot (Figure 8) and the $\log -\log$ plot $(\log$ TV versus $\log t, \log$ FDTV versus $\log \mathrm{t}$, and $\log \mathrm{V}(\mathrm{t})$ versus $\mathrm{V}(\mathrm{t}-\Delta \mathrm{t}))$ are conducted to demonstrate whether REG-I and REG-II are proportional. The figures indicate that these two regions are not symmetrical or equal.

Eliminating the nonlinear part in both REG-I and REG-II shown in Figure 8, we may fit each one of these regions to a straight line. For REG-I, the slope \pm its

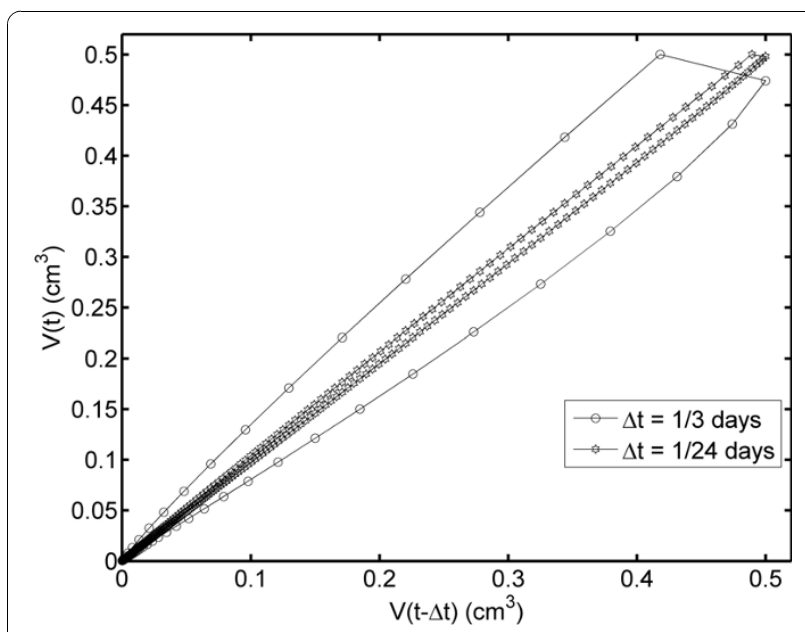

Figure 7 Time consecutive dependence of the TV plot $\left(V^{*}(t)\right.$ vs. $V^{*}(t-\Delta t)$ plot $)$ for $\Delta t=1 / 3$ and $1 / 24$ days. 


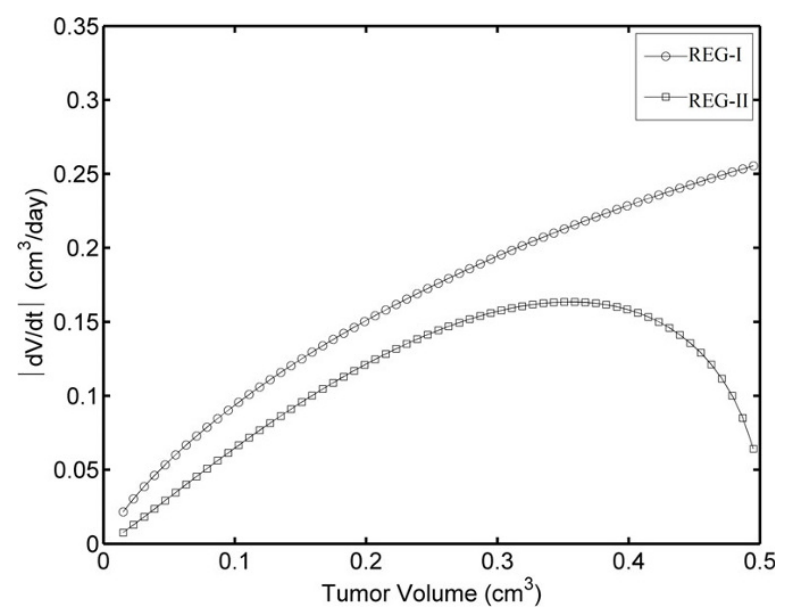

Figure 8 Analysis conducted by separating REG-I and REG-II in a plot of module of FDTV versus TV.

error and the intercept \pm its error are $0.724 \pm 0.011$ and - $0.729 \pm 0.024$, respectively. These respective parameters are $0.992 \pm 0.031$ and $-0.524 \pm 0.068$, for REGII. The ratio between the slopes is 1.37 .

In the CG, the CTV plot shows that $\mathrm{V}(\mathrm{t})$ increases with increasing $V(t-\Delta t)$, as expected. Additionally, the CTV plot for TG2 reveals that both $\mathrm{V}(\mathrm{t})$ and $\mathrm{V}(\mathrm{t}-\Delta \mathrm{t})$ decrease to $0.376 \mathrm{~cm}^{3}$ beyond $V_{0}$. Then, $V(t)$ increases with decreasing $\mathrm{V}(\mathrm{t}-\Delta \mathrm{t})$ until reaching $\mathrm{V}_{\text {min }}$. Beyond this value, both $\mathrm{V}(\mathrm{t})$ and $\mathrm{V}(\mathrm{t}-\Delta \mathrm{t})$ increases.

The patterns shown in Figure 3 and Figure 4 for TG2 are similar to those for TG1. Furthermore, the results obtained for fibrosarcoma Sa-37 TGK are similar to those obtained for Ehrlich tumors in the three experimental groups. For this reason, in the present study, such results are not included.

\section{Discussion}

We show that the macroscopic behavior of both untreated and DEC-treated fibrosarcoma Sa-37 TGK can be realistically modeled using Equation 5. For this, we use previous experimental data for CG, TG1, TG2, and TG3 [14]; the parameters are obtained from fitting these data (Table 1) [17], and both interpolation and extrapolation methods for different time steps $\Delta t(1 ; 1 /$ $3 ; 1 / 8 ; 1 / 24$; and $1 / 48$ days) are used.

\section{Unperturbed fibrosarcoma Sa-37 TGK}

The TV plot corroborates that the complete untreated fibrosarcoma Sa-37 TGK $(i=0)$ exhibits an S shape with three well-defined stages (SI, SII, and SIII) (Figure $1)$. SI is common to each experimental group, and it is associated with the establishment of the tumor in the host. SII is related to rapid tumor growth. SIII of this kinetic shows slow tumor growth and its behavior towards $\mathrm{V}_{\mathrm{f}}$ (asymptotic value).

In SI, $\mathrm{V}_{\mathrm{ob}}$ for fibrosarcoma Sa-37 tumor at 8 days is experimentally observable and palpable but not measurable [14]; however, Equation 5 predicts this value. Nerterets et al. [24], reported tumor diameters below 0.025 $\mathrm{cm}$ via imaging with $\mathrm{X}$-ray phase-contrast micro-CT inline holography. The extrapolation of SI estimates a tumor size of $0.0000082 \mathrm{~cm}^{3}$ to be reached at 7.79 days, with the first approximation assumption that $0.025 \mathrm{~cm}^{3}$ is the smallest volume measured for all tumor types. The differences between these values and those estimated for this tumor type are $0.0000078 \mathrm{~cm}^{3}$ for TV and 0.21 days for time, which are not significant at the experimental level.

Experimentally, TV is measured with a vernier caliper with a precision of $0.005 \mathrm{~cm}$, and the thickness of the mouse skin (between 0.1 and $0.2 \mathrm{~cm}$ ) is taken into account. Our experience indicates that above $0.02 \mathrm{~cm}^{3}$, the mouse skin thickness is negligible as compared with the tumor size [11-14]. Below $0.02 \mathrm{~cm}^{3}$, this thickness is comparable and larger than the tumor size, being more evident when the TV approaches $\mathrm{V}_{\mathrm{ob}}$.

Equation 5 is continuous and smooth for all $\mathrm{t}$ (from $\mathrm{t}=$ 0 up to the end of the experiment), in contrast with the experiment. The tumor sizes are smaller than $10^{-6} \mathrm{~cm}^{3}$, below $\mathrm{V}_{\mathrm{ob}}$, which cannot be observed or measured with any of the current experimental techniques for measuring $\mathrm{TV}$, and therefore, to a first approximation, the sizes are considered as zero, in agreement with our experimental observations [11-14]. This suggests that REG-I consists of two parts: from $t=0$ up to $t_{\mathrm{ob}}\left(\mathrm{t}_{\mathrm{ob}}\right.$ : observable time, in days, for which $\mathrm{V}_{\mathrm{ob}}$ is observed) and from $t_{\mathrm{ob}}$ up to $\tau$. As a result, Equation 5 can be rewritten as

$$
V^{*}(t)= \begin{cases}0 & \text { for } 0 \leq t \leq t_{o b} \\ V_{o} e^{\left(\frac{\alpha}{\beta}\right)\left(1-e^{-\beta(t-\tau)}\right)} & \text { for } t_{o b} \leq t \leq \tau \\ V_{o} e^{\left(\frac{\alpha^{*}}{\beta}\right)\left(1-e^{-\beta(t-\tau)}\right)} & \text { for } \tau \leq t \leq \tau+t^{\prime}\end{cases}
$$

Equation 7 suggests that the MGE is continuous for $t \geq t_{o b}$. Our experience indicates that $V_{o b}$ and $t_{o b}$ depend on the tumor histogenic characteristics, the host type, and the initial concentration of tumor cells inoculated in the host [11-14].

We experimentally observe that fibrosarcoma Sa-37 solid tumors are spheroids between 8 and 10 days (SI of TGK), which are also palpable and observable but not measurable. Our model predicts that TV at 10 days is $0.0025 \mathrm{~cm}^{3}(0.17 \mathrm{~cm}$ in diameter $)$. It is surprising that 
this volume range ( 0.031 to $0.17 \mathrm{~cm}$ in diameter) for which the tumor is spherical coincides with that reported by other authors for the avascular phase $(0.025$ up to $0.2 \mathrm{~cm}$ in diameter) [5,25-32]. Our model estimates that a tumor $0.2 \mathrm{~cm}$ in diameter $\left(0.0042 \mathrm{~cm}^{3}\right)$ is reached at 10.28 days. The differences for volume and time are $0.0017 \mathrm{~cm}^{3}$ and 0.28 days, which are not significant at the experimental level.

The fact that the tumors are spheroids (between 0.000016 and $0.0025 \mathrm{~cm}^{3}$ ) may be explained by a central force field of the Coulomb type due to the fact that the cancer cells are negative charged [33]. It is important keep in mind that a force field is central if and only if it is spherically symmetric. An increase in the tumor cell number occurs when the tumor grows, and as a result, these cells are closer. Since they have the same electrical charge, they are repelled and the tumor is deformed, a fact that explains why the tumor has an ellipsoidal shape after 10 days.

The results show that TGK for SII changes quickly at first (from $V_{\mathrm{s}}$ up to $\mathrm{V}_{\mathrm{i}}$ : concave upwards) and then slowly (from $V_{i}$ up to $V_{i c}$ : concave downwards). This pattern occurs because FDTV first increases and then decreases with increasing TV. In the first case (when both TV and FDTV increase), several factors are involved, such as local growth that is facilitated by enzymes (e.g., proteases) that destroy adjacent tissues and, tumor angiogenesis factors that are produced to promote formation of the vascular supply required for further tumor growth, among others [1]. In the second case (when FDTV decreases with increasing TV), the tumor itself generates different mechanisms that oppose its own growth (i.e., anti-angiogenic substances). If the tumor does not generate such mechanisms, its growth would be exponential and, as a consequence, the tumorhost relationship would be broken, which is not observed in oncological practice [1]. This may indicate that unperturbed tumors intelligently regulate their own growth. This means that the tumor self-organizes, and as a result, new emergent variables appear in order for the tumor to grow, evade the immune system, and achieve maximum survival.

The FDTV behavior may suggest that the tumor doubling time and $\alpha$ are not constant during unperturbed fibrosarcoma Sa-37 TGK, in agreement with Steel [22]. This result is in contrast with the fact that these two kinetic parameters are constant during all TGK, as we assume in this paper and as reported previously by our group [17] and other authors [1,23]. Additionally, the TV dependence of FDTV indicates that, $V_{i}$ may have important implications in DEC planning, if we take into account the fact that the tumor is more sensitive to DEC than healthy tissue [7-15,18-21], the Steel equation [22], and the results of Smith et al. [34].
In SIII, the tumor behavior is explained by the fairly slow rate of growth due to the amount of nutrients and $\mathrm{O}_{2}$ needed for quick expansion of the tumor $[1,22]$.

Both interpolation and extrapolation methods estimate $\mathrm{V}_{\mathrm{m}}, \mathrm{V}_{\mathrm{s}}, \mathrm{V}_{\mathrm{o}}$, and $\mathrm{V}_{\mathrm{f}}$ with good accuracy as well as their respective times, which are experimentally observed [14]. This is reasonable because the differences between the experimental and theoretically predicted values for these volumes and times at the experimental level are not significant. Furthermore, these methods predict $V_{i}$ and $V_{\text {ic }}$ and their respective times, which are not available from a TV plot. These points may have important implications in TGK and tumor treatment. The existence of $V_{\text {ic }}$ establishes the irreversibility of TGK.

Our experience in preclinical studies indicates that a good DEC effectiveness is obtained for TV smaller than $1.5 \mathrm{~cm}^{3}[11,12,14]$; however, it markedly decreases for TV bigger than $1.5 \mathrm{~cm}^{3}$ although DEC treatment is repeated several times [13]. In clinical studies, DEC effectiveness decreases when TV $\geq 8 \mathrm{~cm}^{3}[9,10,15]$. It is interesting that $1.5 \mathrm{~cm}^{3}$ is near to $V_{i}$, fact that may suggest that DEC treatment is effective for TV below $V_{i}$, indicating that is important to know this TV in TGK. $V_{i}$ may be a criterion of application for this therapy. We suggest to apply electrotherapy for TV below $V_{i}$.

\section{DEC-treated fibrosarcoma Sa-37 TGK for TG2}

In TG2, REG-IIa (from $V_{o}$ up to $V_{\text {min }}$ ) is related to the rapid tumor inhibition resulting from DEC cytotoxic action, and REG-IIb (from $V_{\text {min }}$ up to $V_{f}$ ) represents the tumor prevalence (tumor re-growth). However, FDTVTV plot reveals that FDTV first decreases up to FDTV $_{\text {min }}$ and then increases with decreasing TV in REG-IIa. This may suggest that in this region the tumor self-organizes whereas its volume decreases, indicating that DEC dose is not effective, an aspect not addressed in the literature. As a result, FDTV tends to 0.000068 $\mathrm{cm}^{3} /$ days corresponding to $\mathrm{V}_{\mathrm{min}}$, from which TGK triggers.

Tumor destruction (when both TV and FDTV decrease) is caused by DEC cytotoxic action, which induces toxic products in the tumor, generated by electrochemical reactions [19], and it potentiates humoral and cellular components of the immune system [20]. At this time interval, necrosis, apoptosis, chronic inflammation, polymorphous nuclear, monocytes, vascular congestion, and the activation of macrophages and $\mathrm{T}$ lymphocytes have been observed [7-15,18-21].

Tumor self-organization is not observed in the TV plot and occurs when FDTV changes of slope independently of the decrease of TV. This timing may occur because the DEC dose used does not induce significant damage to the tumor. As a result, the tumor potentiates its existing mechanisms and/or generates other new 
mechanisms for its own protection, growth, and metastasis processes in order to reach its maximum survival. This second process can also be explained from the point of view of the complexity theory because the tumor is self-organized and new emergent variables appear [35-38]. This self-organization process of the tumor dominates the process of tumor destruction caused by DEC action, with TV reaching $\mathrm{V}_{\min }$ and consequential tumor re-growth (REG-IIb).

$\mathrm{V}_{\min }$ observed in the TV plot for TG2 is very important from a therapeutic point of view because when TV reaches this value, DEC should be repeated $[9,10,13]$; however, the results shown in this study indicate that the tumor is self-organized when it reaches $\mathrm{V}_{\text {min }}$. For us, the existence of $\mathrm{FDTV}_{\min }$ (corresponding to $\mathrm{TV}=$ $0.376 \mathrm{~cm}^{3}$ ) on the FDTV-TV plot is surprising because this tumor self-organization process is not observed in the TV plot and therefore its explanation is not possible from this plot. This is relevant, at the therapeutic level because DEC stimulus alone or combined should be repeated when the TV reaches this value.

This procedure may be implemented in practice through two possible ways: 1 ) by weekly measuring (once or twice) the TV during the first three months after DEC treatment by means of a vernier caliper (for superficial tumors) or ultrasound (for visceral tumors) and 2) by knowing the tumor relaxation time $\left(\mathrm{T}_{\mathrm{rt}}\right)$ of a small sample treated with DEC by means of Nuclear Magnetic Resonance method.

In the first way, we observe a significant decrease of TV in DEC treated patients during the first three months, after this time, a tumor re-growth is observed if the dose is not effective [15]. We suggest two measurements/week of TV to obtain various experimental points in the first three months of observation so that the values of the parameters: $\alpha, \beta, \gamma$, and $i_{o}$ can be calculated knowing the values of $\mathrm{V}_{\mathrm{o}}$ and $\mathrm{TV}$ on the first four measurements. Then, a numerical method is used to solve a non-homogeneous system of four non-linear equations with these four unknown parameters. This is possible because MGE has a good prediction capability to describe both unperturbed and perturbed tumor growths [17]. We can predict the temporal behavior of TV (TV plot) and of its derived (FDTV plot) once the values of these four parameters are well-known and then estimate FDTV $_{\min }$ in a FDTV-TV plot. If FDTV changes the sign of its slope (positive to negative) although TV continues increasing, we suggest to repeat this therapy and/or to combine it with another therapeutic procedure, as shown in this study. Therefore, we do not recommend the use Tomography Axial Computerized and Imaging Nuclear Magnetic Resonance, because of their high costs and the regulatory norms established for the use of each one of these imaging techniques.
In the second way, the knowledge of $\mathrm{T}_{\mathrm{rt}}$ is important because we know the time for which the tumor recovers after DEC treatment, and the times that DEC treatment should be repeated in order to the tumor is not self-organize (for example, at a time smaller than $\mathrm{T}_{\mathrm{rt}}$ ). The knowledge concerning to these two facts will allow us to determine the exact time at which the DEC should be repeated, and as a result, it will allowed one to avoid unnecessary DEC stimulus to the patient. The tumor self-organization process is slower if the duration of the DEC cytotoxic effect induced into the tumor is greater than $\mathrm{T}_{\mathrm{rt}}$. In a previous study, we corroborate theoretically that DEC effectiveness increases with the increase of the duration of DEC cytotoxic effect induced into the tumor [17]. The introduction of any of these two possible ways in our experiments will lead to a high antitumor effectiveness, which suggests that our future researchers should take this fact into account.

\section{DEC-treated fibrosarcoma Sa-37 TGK for TG3}

In TG3, REG-IIc (when TV and FDTV both rapidly decrease) may be explained from a biological point of view by DEC cytotoxic action, as we propose above. It should be noted that in the FDTV-TV plot, just before the tumor reaches $V_{d}$, there is a change of slope for FDTV with $V_{\text {id }}$, implicating that other antitumor mechanisms have been activated (e.g., the activation of cellular and humoral components of the immune system mentioned above and others unreported until now). In contrast to TG2, in TG3, this change of slope for FDTV does not change its negative sign between $V_{o}$ and $V_{d}$.

The net rate of the antitumor processes involved between $V_{o}$ and $V_{i d}$ is higher than that resulting from other antitumor processes induced between $V_{\text {id }}$ and $V_{d}$. From a biophysical point of view, this indicates the existence of at least two other unknown main antitumor mechanisms, which can occur simultaneously. Each one of these mechanisms has its own time constant, in agreement with previous reports [11]. As a result of these antitumor mechanisms, the tumor is completely destroyed (or reversible). This is corroborated, as TV and FDTV tend to zero when TV is smaller than $V_{d}$; in agreement with our results [17].

The fact that the complete TGK for TG3 is a closed loop suggests the reversibility of the tumor. We believe that this is true if TV is comprehended between $V_{m}$ and $\mathrm{V}_{\mathrm{i}}$. This fact corroborates the above discussion regarding the goal of $V_{\text {id }}$ in DEC treatment. Some additional experiments are required to prove this statement.

This loop shows that REG-I of TGK (before DEC treatment) and REG-II (after DEC treatment) are asymmetric for all $\Delta t$ values. The linear fits of these two regions suggest that the slope of the curve for REG-II is 1.37 times higher than that for REG-I, a fact that 
corroborates that the TV regression rate is proportional to the rate of growth, in agreement with the NortonSimon hypothesis [39]. Prior to this study, we think that these rates are equal.

This new paradigm forces us to reconsider our knowledge and to modify our traditional approach to research and treatment. This statement is relevant for ET because it completely changes the conception of cancer treatment. The actual idea behind in vitro and in vivo studies is to treat the tumor and then to observe its evolution, which is not known to priori [7-15,18-21]. However, the existence of $V_{d}$ establishes that fibrosarcoma Sa-37 tumors have a DEC threshold for which the tumor is completely destroyed, as demonstrated experimentally and theoretically for Ehrlich and fibrosarcoma Sa-37 tumors [14,17], in agreement with other studies $[9,10,18,21,33]$. This is possible if we establish an explicit dependence of $V_{d}$ as a function of the parameters of Equation 7, the host type, and the ET parameters (dosage and exposure time of DEC, electrode array, and times that DEC is repeated). This is very complex at the experimental and theoretical levels; however, mathematical modeling may be a useful tool for finding an approximate solution (analytical or numerical) to this problem. Such modeling will lead to further improvement in the treatment of solid tumors, and it can also help guide treatment decisions for therapists treating patients (or animals) with this disease. In addition, this statement will contribute to standardizing this therapy.

\section{New predictions and hypothesis for TGK}

In the physical sciences, mathematical theory and experimental investigation have always worked together. Mathematical theory can help to direct experimental research, while the results of experiments help to refine the modeling [2]. This is precisely one of the intentions of this manuscript.

Although Equation 5 (or 7) does not reveal other information, we can propose hypothesis-testing (or hypothesis-generating) methods from it and our experimental observations. The fact that in REG-I of TGK, specifically in SI, for all experimental groups, Ehrlich and fibrosarcoma Sa-37 solid tumors are not observed below $\mathrm{V}_{\mathrm{ob}}$ (tumor cells in suspension) and are observed above $\mathrm{V}_{\mathrm{ob}}$ (solid tumor or tumor mass) may suggest the existence of a phase transition. It is more evident for these tumor types in REG-II of TGK for TG3 when the solid tumor passes from its active phase (below $V_{d}$ ) to the phase in which the tumor is completely destroyed due to DEC action (above $V_{d}$ ) [14]. In both cases, these transitions are named $\mathrm{PT}_{1}$ and $\mathrm{PT}_{2}$, respectively, as schematically represented in Figure 9 and Figure 10.

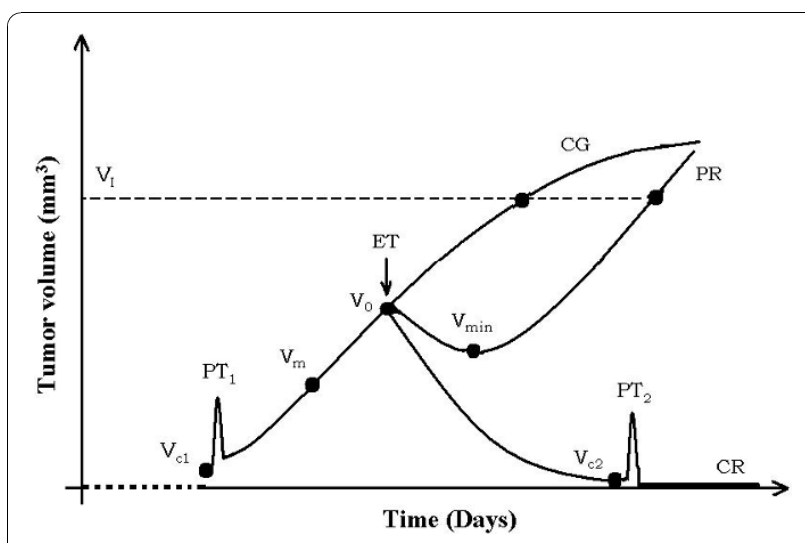

Figure 9 Schematic representation of TGK.

This is also supported if we remember that a phase transition has the characteristic of taking a medium with given properties and transforming some (or all) of it into a new medium with new properties (i.e., the transformation of a thermodynamic system from one phase to another) $[40,41]$.

We know from thermodynamics that at the phase transition point, physical properties may undergo abrupt changes: for instance, the volumes of the two phases may be vastly different, as observed in SI (below and after $V_{\mathrm{ob}}$ ) and REG-II in TG3 (below after $\mathrm{V}_{\mathrm{d}}$ ), a fact that could suggest the existence of a critical TV in SI, $\mathrm{V}_{\mathrm{cl}}$, and another in REG-II in TG3, $\mathrm{V}_{\mathrm{c} 2}$, as schematically represented in Figure 9 and Figure 10. We believe that when $V_{c 1}\left(V_{c 2}\right)$ is reached; the tumor begins to grow (completely destroyed).

It is possible that such a phase transition involves a large amount of energy (a dissipative system) accompanied by fluctuations, chaos, and/or self-organization processes with the presence of emergent variables, in agreement with other authors [5,35-38,40-46].

Several authors have reported various phenomena that occur in SI of TGK, such as: a transition from the tumor avascular phase to the vascular phase (angiogenesis), which is accompanied by fluctuations [5,25-28,31,32]; the existence of a stochastic transition at the change between these two tumor phases [29]; the disruption of normal blood vessels of the organs in which the tumor is developing caused by chaotic growth [25]; the existence of a threshold under which sprouts cannot reach the tumor during the growth of the vascular network [46]; among others. It is interesting that our model reveals that SI is highly non linear, a fact that could be associated with the presence of chaos [5,42,43], in agreement with other authors $[5,42,43]$. This corresponds with established non-equilibrium thermodynamics, in which systems driven out of equilibrium (as 


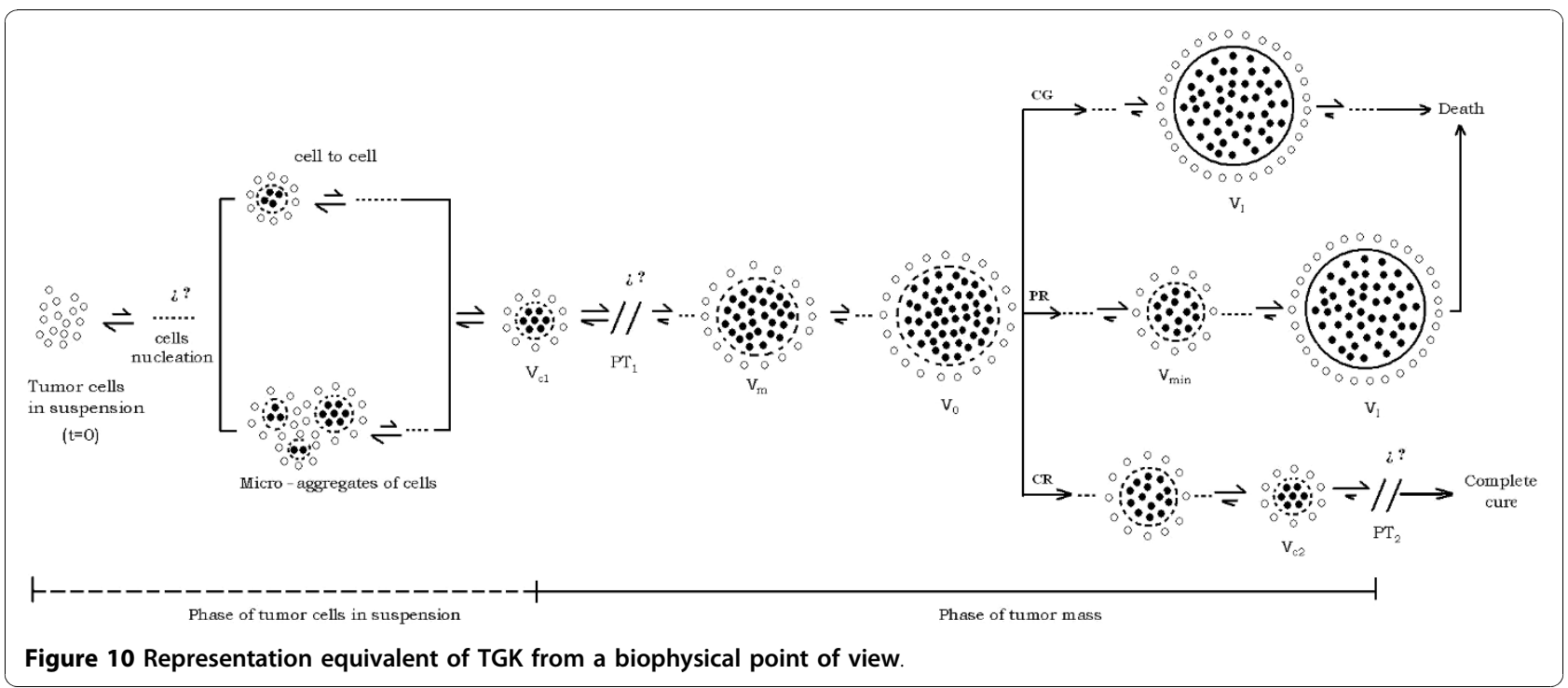

solid tumors and biological systems generally are) often exhibit fluctuations or phase transitions [35,44]. In addition, these systems can develop from disorder (systems known as dissipative) because they are formed and maintained by dissipative processes that take place due to an exchange of energy and matter between the system and its environment, and they disappear if that exchange ceases. From Equation 1 (5 or 6 ), it may be corroborated that a tumor is a dissipative system because $i_{o}$ is much lower than $i$ [17]. The biological processes that are constantly receiving, transforming, and dissipating chemical energy can, and do, exhibit properties of self-organization far from thermodynamic equilibrium $[35-38,44,45]$.

MGE offers information of the global dynamics of unperturbed and DEC treated tumors and therefore only gives a limited understanding about the self-organization processes in TGK. However, we believe that these processes are involved in unperturbed and DEC treated TGK for discussed above and the following facts, which are implicitly in MGE: 1) Self-organization makes sense only in relation to the whole: it is the whole that selforganizes into a multitude of interacting levels. At the same time, the whole cannot sustain its integrity, if the process of self-organization does not work. This suggests that self-organization has an important role in the formation, maintenance, and function of cells, tissues, organs and the complete human body. 2) A key requirement for a self-organizing system is nonlinearity and therefore the self-organizing systems are governed by nonlinear dynamics [47], in agreement with our results. 3) Gompertzian dynamics emerges as a result of the fractal-stochastic dualism, which is a universal natural law of biological complexity [48], in agreement with Brú et al.
[49]. 3) System changes from non-order to order, from low-grade order to advanced order, basis on the principle of auto-organization adaption [50]. 4) Cancer is a reflection of a failing system; preventive steps should involve rebalancing the entire system through lowering of disorderly complexity, entropy, and optimizing self-organization with orderly complexity [51]. 5) The malignant tumor is a complex system and therefore this complexity expresses its functionality and reflects a high degree of resilience and robustness to environmental challenges through their self-adaptation and internal self-organization [51]. 6) The process of tumor cell growth, invasion and metastasis involves a self-organized cascade of multiple tumor-host and tumor-immune interactions [52]. Self-organization might be a general principle in cellular organization and an elegant, efficient way to optimally organize cellular structures [53]. 7) Self-organization occurs when a real system evolves toward a higher differentiation from its initial state (or pre-system phase) [51]. These two phases are revealed with MGE: pre-tumor phase (below $\mathrm{V}_{\mathrm{ob}}$ ) and solid tumor phase (above $\mathrm{V}_{\mathrm{ob}}$ ). Also, this differentiation is observed in our pathological studies [11-15], and it is the cause of the aggressiveness and difference in the cellular/molecular patterns of the different types of malignant tumors [1]. In spite of these facts and others, more studies at cellular/molecular/ atomic/quantum levels and new physic-mathematical approaches are needed to have more meaningful results about the self-organization process in TGK.

During such a phase transition, a tumor either absorbs or releases a fixed (and typically large) amount of energy, which is characteristic of a first-order phase transition. Because energy cannot be instantaneously transferred between the tumor and it's surrounding 
healthy tissue, first-order transitions are associated with "mixed-phase regimes" in which some parts of the system have completed the transition and others have not. Based on statistical physics, mixed-phase systems are difficult to study, because their dynamics are violent and challenging to control [40].

The hypotheses proposed in this study can doubtlessly be seriously attacked by many; however, this study sets the basis to derive some practical understanding from our diverse (and often, at this time, empirical) experimental and clinical observations in cancer electrotherapy. The availability of powerful computers has already helped to bridge the gap between observations and predictions in many complex problems, and a few attempts have already been made to attack the problem of tumor growth with mathematical models.

We are recognizing biophysics principles that may be broadly applied in developing more useful programs of DEC treatment of solid tumors. To begin to understand the complexity of the proposed system, novel simulations must be developed, incorporating concepts from many scientific areas such as cancer research, statistical mechanics, applied mathematics, and nonlinear dynamical systems.

Our results suggest that the MGE should be modified, or a new mathematical approach should be proposed in order to describe TGK and explain the presence of at least one of these phenomena. These results are, in agreement with Bellomo et al. [2], who proposed that "future research will definitely refine and improve the existing models, while the analysis of the inherent mathematical problems will hopefully lead to new mathematics, allowing us to tackle problems presently beyond our technical abilities".

\section{Conclusion}

In conclusion, the modified Gompertz equation is likely to lead to insights within cancer research. Such insights hold promise for increasing our understanding of tumors as self-organizing systems and, the possible existence of phase transitions in tumor growth kinetics, which, in turn, may have a significant impact both on cancer research and on clinical practice.

\section{List of abbreviations used}

ET: electrotherapy; DEC: direct electric current; CG: control group; TG1: treated group 1; TG2: treated group 2; TG3: treated group 3; MGE: modified Gompertz equation; TV: tumor volume; TGK: tumor growth kinetics; REG-I: part of TGK before DEC treatment; REG-II: part of TGK after DEC treatment; REG-lla and REG-Ilb: sub-regions of REG-II for TG2; REG-Ilc and REG-Ild: subregions of REG-II for TG3; FDTV: first derivative of tumor volume; FDTV max: maximum FDTV observed in TGK for the CG; FDTV min: minimum FDTV observed in TG2; TV plot: tumor volume versus t plot; FDTV plot: time dependence of first derivative of tumor volume plot; FDTV-TV plot: first derivative of tumor volume versus tumor volume; CTV plot: time consecutive dependence of tumor volume plot; $V_{0}$ : initial volume at which DEC is supplied; $V_{\mathrm{ob}}$ : first non-zero value of $\mathrm{TV} ; \boldsymbol{\tau}$ : time delay; $\mathrm{T}_{\mathrm{rt}}$ : tumor relaxation time; $V_{\text {min }}$ : minimum $T V_{i} V_{d}$ : TV from which begins tumor complete destruction; $V_{\text {id }}$ : inflection point in REG- $\|_{c} ; V_{m}$ : smallest measurable TV; $V_{f}$ : final volume of TGK; $t_{o b}$ : time at which $V_{o b}$ is observed; $V_{i}$ : point of inflection in TGK; $V_{S}$ : TV that separates SI and SII; $V_{i c}$ : TV that separates SII and SIII; $\Delta$ t: time step; SI, SII, and SIII are the first, second, and third stages in TGK of the control group, respectively; $\mathrm{PT}_{1}$ : phase transition between the phases of tumor cells in suspension and a solid tumor; $V_{c 1}$ : critical volume for which $\mathrm{PT}_{1}$ occurs; $\mathrm{PT}_{2}$ : phase transition between an active solid tumor and a completely destroyed tumor; $\mathrm{V}_{c 2}$ : critical volume for which $\mathrm{PT}_{2}$ occurs.

\section{Acknowledgements}

The authors wish to thank Emilio Suárez and Dr. José Luis García Cuevas for their technical assistance. JJGN acknowledges the support of Dr. Isaac Hernández Calderón. This research was supported by Physics Department, Research Center and Advanced Studies of National Polytechnic Institute, México and the Ministry of Superior Education, Republic of Cuba. Also, we thank in a special way the reviewers for their invaluable recommendations and suggestions.

\section{Author details}

${ }^{1}$ Universidad de Oriente, Centro Nacional de Electromagnetismo Aplicado, Departamento de Bioelectromagnetismo, Grupo de Bioelectricidad, Av. Las Américas s/n. G.P. 4078. Santiago de Cuba 90400, Cuba. ${ }^{2}$ Departamento de Física, Centro de Investigación y Estudios Avanzados del Instituto Politécnico Nacional, Laboratorio de Estimulación Magnética, Av. Instituto Politécnico Nacional 2508, Col. San Pedro Zacatenco, Ap. Post. 14-740, México, D.F. 07000, México 07360, Distrito Federal, México. ${ }^{3}$ Universidad de Oriente, Centro de Biofísica Médica, Departamento de Biofísica. Santiago de Cuba 90500, Cuba. ${ }^{4}$ Universidad de Oriente, Facultad de Ciencias Naturales, Departamento de Física. Calle Patricio Lumumba s/n. Santiago de Cuba 90500, Cuba. ${ }^{5}$ Universidad de Oriente, Facultad de Ciencias Naturales, Departamento de Farmacia. Patricio Lumumba s/n. Santiago de Cuba 90500, Cuba. ${ }^{6}$ Hospital Infantil Sur, Servicio de Oncohematología. Santiago de Cuba 90200, Cuba. ${ }^{7}$ Dirección Municipal de Salud Pública. Servicio de Genética. Santiago de Cuba 90500. Cuba. ${ }^{8}$ Hospital Oncológico Conrado Benítez, Servicio de Mastología. Santiago de Cuba 90500, Cuba. ${ }^{9}$ Hospital Provincial Saturnino Lora, Servicio de medicina Interna. Santiago de Cuba 90500, Cuba. ${ }^{10}$ Instituto Finley. Ave. 27 No. 19805, La Lisa, A.P. 16017 Cod. 11600. La Habana.

\section{Authors' contributions}

LEBC planned the study, and participated in its design and coordination, and also assisted with the manuscript. JJGN participated in its design and discussion, and also contributed to the manuscript. ARA and JAGJ organized this study and participated in the elaboration of the software to obtain the results shown. HMCC, MMG, MFS, MVJ, TRG, MAOM, SCAB, FSP, LZO, MCCQ, SES, VCC, IBC, and GSG participated in the design of this study, and contributed to the analysis, interpretation, and discussion of the results. All authors read and approved the final manuscript.

It is important to point out that studies in cancer necessarily require the knowledge of a multidisciplinary group of researchers because cancer is not well-understand, and currently, no therapy that completely cures this illness has been reported.

\section{Competing interests}

The authors declare that they have no competing interests.

Received: 6 November 2009 Accepted: 28 October 2010 Published: 28 October 2010

\section{References}

1. Cotran RS, Kumar V, Collins T: Patología Estructural y Funcional. Sexta Edición. McGraw-Hill- Interamericana de España, S.A. U Madrid; 1999, 277-347.

2. Bellomo N, Li NK, Maini PK: On the foundations of cancer modeling: selected topics, speculations, and perspectives. Math Mod Meth Appl Sci 2008, 18:593-646.

3. Stein WD, Figg WD, Dahut W, Stein AD, Hoshen MB, Price D, Bates SE, Fojo T: Tumor Growth Rates Derived from Data for Patients in a Clinical Trial Correlate Strongly with Patient Survival: A Novel Strategy for Evaluation of Clinical Trial Data. The Oncologist 2008, 13:1046-1054. 
4. González MJ, Herrera FL, Miranda-Massari JM, Guzmán AM, Riordan NH, Ricart CM: Tumor growth parameters of in-vivo human breast carcinoma: a proposed mathematical model for tumor growth kinetics. $P R$ Health Sci J 2006, 25:71-73.

5. Preziosi L: Cancer Modeling and Simulation. Chapman \& Hall/CRC Mathematical Biology and Medicine Series 2003.

6. Drasdo D, Höhme S: A single-cell-based model of tumor growth in vitro: monolayers and spheroids. Phys Biol 2005, 2:133-147.

7. Veiga VF, Nimrichter L, Teixeira CA, Morales MM, Alviano CS, Rodrigues ML, Holandino C: Exposure of human leukemic cells to direct electric current: generation of toxic compounds inducing cell death by different mechanisms. Cell Biochem Biophys 2005, 42:61-74.

8. Wartenberg M, Wirtz N, Grob A, Niedermeier W, Hescheler J, Peters SC, Sauer H: Direct current electrical fields induce apoptosis in oral mucosa cancer cells by NADPH oxidase-derived reactive oxygen species. Bioelectromagnetics 2008, 29:47-54.

9. Kuan-hong Q: A controlled study of electrochemical therapy and electrochemical treatment compounded resection in 106 patients with malignant tumor in body surface. Proceedings of the 8th Congress on Tumors of International Association of Biological Closed Electric Circuits, Nanning, China 2004, 112-113.

10. Yuling X, Chaoyang L, Deruo I, Wei Z: Clinical effectiveness of treatment of superficial tumors with electrochemical therapy (EChT). Proceedings of the 8th Congress on Tumors of International Association of Biological Closed Electric Circuits, Nanning, China 2004, 133-139.

11. Cabrales LEB, Ciria HMC, Bruzón RNP, Quevedo MCS, Aldana RH, González LMO, Salas MF, Peña OG: Electrochemical treatment of mouse Ehrlich tumor with direct electric current. Bioelectromagnetics 2001, 22:316-322.

12. Cabrales LEB, Salas MF, Peña OG, Fernández SG, Brooks SA: Efectos de la CED en el crecimiento de un Fibrosarcoma Sa-37 en dos hospederos BALB/C y C57BL/6. MEDISAN 2002, 1:36-40.

13. Cabrales LEB, Aldana RH, Bruzón RNP, Montes de Oca L, Ciria HMC, Lafargue AL, Quevedo MCS, Toirac RR, Reyes AF: Efectos de la corriente eléctrica directa en el tumor murino subcutáneo de Ehrlich. I: Estudios de necrosis y volumen del tumor en estadíos avanzados. Rev Bras Cancerol 1998, 44:203-210.

14. Ciria HMC, Quevedo MS, Cabrales LEB, Bruzón RNP, Salas MF, Peña OG, González TR, López DS, Flores M: Antitumor effectiveness of different amounts of electrical charge in Ehrlich and fibrosarcoma Sa-37 tumors. BMC-Cancer 2004, 4:87-91.

15. Jarque MV, Mateus MAO'F, Palencia FS, Cabrales LEB, Jing-Hong L, Ciria HMC, Brooks SCA, Salas MF, Quevedo MCS, Bestard NR, Fernández SG, González TR, Hernández MR, Bordelois KC: First clinical experiences in Cuba over the use of electrotherapy in four patients with superficial malignant solid tumors. Rev MEDISAM 2007, 11:1-8.

16. Aguilera AR, Cabrales LEB, Ciria HMC, Pérez YS, Oria ER, Brooks SA: Distributions of the potential and electric field of an electrode elliptic array used in electrotherapy: Analytical and numerical solutions. Math Comput Simul 2008, 79:2091-2105.

17. Cabrales LEB, Aguilera AR, Jiménez RP, Jarque MV, Ciria HMC, Reyes JB, Mateus MAO, Palencia FS, Ávila MG: Mathematical modeling of tumor growth in mice following low-level direct electric current. Math Comput Simul 2008, 78:112-120

18. Ren RL, Vora N, Yang F, Longmate J, Wang W, Sun H, Li JR, Weiss L, Staud C, McDougall JA, Chou CK: Variations of dose and electrode spacing for rat breast cancer electrochemical treatment. Bioelectromagnetics 2001, 22:205-211.

19. Li KH, Xin YL, Gu YN, Xu BL, Fan DJ, Ni BF: Effects of Direct Current on Dog Liver: Possible Mechanisms for Tumor Electrochemical Treatment. Bioelectromagnetics 1997, 18:2-7.

20. Miklavčič $D, A n D$, Belehradek J Jr, Mir LM: Hot's immune response in electrotherapy of murine tumors by direct current. Eur Cytokine Netw 1997, 8:275-279

21. Parise O, Oliveira LO, Telló M, Gioso MA, Buzaid AC: Electrolysis and Electrochemical Therapy in the Treatment of Spontaneous Head and Neck Tumors of Cats and Dogs. Annual Meeting \& Research Workshop on the Biology, Prevention and Treatment of Head and Neck Cancer 2006.

22. Steel GG: Growth Kinetics of Tumors. Clarendon Press, Oxford; 1977.

23. Vaidya VG, Alexandro FJ: Evaluation of some mathematical models for tumor growth. J Biomed Comput 1982, 13:19-36.
24. Nesterets $Y$, Gureyev T, Stevenson A, Pogany A, Wilkins S, Kincaid R, Ye H, Vogelsang L, Lipson E, Coman I, Fourmaux S, Kieffer JC, Krol A: Soft tissue small avascular tumor imaging with $\mathrm{x}$-ray phase-contrast micro-CT inline holography. In Medical Imaging 2008: Physics of Medical Imaging. Proceedings of the SPIE Edited by: Hsieh, Jiang; Samei, Ehsan 2008, 6913:69133Z-69133Z-7.

25. Goldman E: The growth of malignant disease in man and the lower animals with special reference to the vascular system. Lancet 1907, ii:1236-1240.

26. Hogea CS, Murray BT, Sethian JA: Simulating Complex Tumor Dynamics from Avascular to Vascular Growth using a General Level Set Method. Mathematical Biology 2005, 53:1-5.

27. Roose T, Chapman SJ, Maini PK: Mathematical Models of Avascular Tumor Growth. SIAM Review 2007, 49:179-208.

28. Jiang Y: A Multiscale Model for Avascular Tumor Growth. Biophysical Journal 2009, 89:3884-3894

29. Mohammadi B, Haghpanah V, Larijani B: A stochastic model of tumor angiogenesis. Computers in Biology and Medicine 2009, 38:1007-1011.

30. Sundareshan MK, Fundakowski R: On the Equivalence of Mathematical Models For Cell Proliferation Kinetics. Cell Proliferation 2008, 17:609-618.

31. Komarova NL: Mathematical modeling of tumorigenesis: mission possible. Cancer biology. Current Opinion in Oncology 2005, 17:39-43.

32. Ribatti D, Vacca A, Dammacco F: The Role of the Vascular Phase in Solid Tumor Growth: A Historical Review. Neoplasia 1999, 1:293-302.

33. Haltiwanger S: The Electrical Properties of Cancer Cells.[http://www. royalrife.com/haltiwanger1.pdf], [Last update: April 2nd 2008].

34. Smith DG, Potter SR, Lee BR, Ko WW, Drummond WR, Telford JK, Partin AW: In vivo measurement of tumor conductiveness with the magnetic bioimpedance method. IEEE Trans Biomed Eng 2000, 47:1403-1405.

35. Nicolis G, Prigogine I: Self Organization in Non-Equilibrium Systems. J Wiley and Sons New York; 1977, Chaps. III and IV.

36. Jensen HJ: Self-Organized Criticality: Emergent Complex Behavior in Physical and Biological Systems. Cambridge Lecture Notes in Physics 10, Cambridge University Press; 1998.

37. Camazine S, Deneubourg JL, Franks NR, Sneyd J, Theraulaz G, Bonabeau E: Self-Organization in Biological Systems. Princeton University Press; 2003.

38. Corning PA: The Re-Emergence of "Emergence": A Venerable Concept in Search of a Theory. Complexity 2002, 7:18-30.

39. Norton L: Conceptual and practical implications of breast tissue geometry: toward a more effective, less toxic therapy. Oncologist 2005, 10:370-381 [http://www.TheOncologist.com/cgi/content/full/10/6/370], last update: February 9, 2007.

40. Landau LD, Lifshitz EM: Statistical Physics. In Part 1, of Course of Theoretical Physics. Volume 5. 3 edition. Pergamon; 1994.

41. Patashinskii AZ, Pokrovskii VL: Fluctuation Theory of Phase Transitions. [in Russian], Nauka, Moscow; 1982.

42. Devaney RL: An Introduction to Chaotic Dynamical Systems. Westview Pressi 22003.

43. Werndl C: What are the New Implications of Chaos for Unpredictability? The British Journal for the Philosophy of Science 2009, 60:195-220.

44. Glansdorff P, Prigogine I: Thermodynamic Theory of Structure Stability and Fluctuations. Wiley and Sons, London; 1971.

45. Brogliato B, Lozano R, Maschke B, Egeland O: Dissipative Systems Analysis and Control. Theory and Applications. Springer Verlag, London; 22007.

46. Amyot F, Small A, Boukari H, Camphausen K, Gandjbakhche A: Topology of the heterogeneous nature of the extracellular matrix on stochastic modeling of tumor-induced angiogenesis. Microvascular Research 2009, 77:87-95.

47. Kurtzweil R: The singularity is near. New York, USA: Penguin Group; 2005, 90-155.

48. Waliszewski P, Konarski J: A Mystery of the Gompertz Function. In Fractals in Biology and Medicine. Edited by: Losa GA, Merlini D, Nonnenmacher TF and Weibel ER. Birkhäuser Basel. Mathematics and Biosciences in Interaction Books; 2005:277-286.

49. Brú A, Albertos S, Subiza JL, García-Asenjo JL, Brú I: The Universal Dynamics of Tumor Growth. Biophysical Journal 2003, 85:2948-2961

50. Guntern G: Auto-organization in human systems. Behavioral Science 2007. 27:323-337.

51. Janecka IP: Cancer control through principles of systems science, complexity, and chaos theory: A model. Int J Med Sci 2007, 4:164-173. 
52. Kraus $\mathrm{M}$, Severin $\mathrm{T}$, Wolf $\mathrm{B}$ : Relevance of microenvironmental $\mathrm{pH}$ for selforganized tumor growth and invasion. Anticancer Res 1994, 14:1573-1583.

53. Misteli T: The concept of self-organization in cellular architecture. $J$ Cell Biol 2001, 155:181-186.

\section{Pre-publication history}

The pre-publication history for this paper can be accessed here: http://www.biomedcentral.com/1471-2407/10/589/prepub

doi:10.1186/1471-2407-10-589

Cite this article as: Cabrales et al:: Modified Gompertz equation for electrotherapy murine tumor growth kinetics: predictions and new hypotheses. BMC Cancer 2010 10:589.

Submit your next manuscript to BioMed Central and take full advantage of:

- Convenient online submission

- Thorough peer review

- No space constraints or color figure charges

- Immediate publication on acceptance

- Inclusion in PubMed, CAS, Scopus and Google Scholar

- Research which is freely available for redistribution

Submit your manuscript at www.biomedcentral.com/submit
C Biomed Central 